$$
\mu+\mu^{4}+\mu^{5}=1-\mu^{4} \simeq 0.854 .
$$

It seems unlikely that one can reach every position (below $H$ ) which has value less than 1 , but I don't know of a counterexample.

2. A similar calculation reveals that for the analogous problem in three dimensions it is impossible for a peg to reach the eighth row. The seventh row can be reached, however.

\section{Phil Rippon}

open University.

Milton Keynes.

\section{CONFERENCE REPORT}

\section{GROUPS IN GALWAY 10-11 MAY 1985}

A Conference on Group Theory, sponsored by University College, Calway, the Royal Irish Academy, and the Irish Mathematical Society was again held at University College, Calway, on Friday and Saturday 10 th and 11 th May 1985. The invited speakers were T.O. Hawkes (Warwick), G.D. James (Cambridge), T.J. Laffey (UCD), P.D. MacHale (UCC), T.G. Murphy (TCD), and S.J. Tobin (UCG). Lectures were also given by D.W. Lewis (UCD) and M. ó'Searcóid (UCC).

Seán Tobin opened the Conference with his talk entitled 'Razmyslov Algebras' (see I.M.S. Newsletter 13 (March 1985) pp. 57-65). He explained Razmyslov's construction of a nonsoluble group of exponent $p^{2}$, for odd primes $p$.

Tim Murphy, in his lecture on 'Tensor Groups', discussed the duality between linear groups and the sets of tensors which they $f i x$. He also illustrated the use of Penrose's notation for tensors, and his hand-out demonstrated the qualities of the TEX computer typesetting system.

Gordon James described 'A q-Analogue of the Symmetric Group Algebra'. Given a field $F$ of characteristic $p$, and a nonzero element $q$ of $F$, he defined an $F$-algebra $H$, whose properties (for example the number of its simple right ideals, and the dimension of its centre) can be obtained from those of the group algebra over $F$ of the symmetric group by substituting $q$ for $p$.

Tom Laffey's title was 'Some Maximal Subgroups of the General Linear Group'. He began by presenting a short proof (due to Radjavi) of the fact that every element of $S L_{n}(F)$ is a commutator in $G L_{n}(F)$, except when $n=|F|=2$. He then proved that the invertible monomial matrices form a maximal subgroup 
of $G L_{n}(F)$, provided $|F|>5$, and he showed how this, and related results, have been used to classify certain linear maps from a space of square matrices to itself.

Des Macthale spoke on 'The Relationship Between $|G|$ and |Aut G| for a Finite Group'. He reported on functions $f(n)$ such that if $p^{f(n)}$ divides $|G|$, then $p^{n}$ divides $|A u t G|$, and also on results related to the conjecture that if $G$ is a noncyclic p-group of order $p^{3}$ or more, then $|G|$ divides |Aut $G \mid$. He finished by mentioning a number of other open questions.

In the last lecture of the Conference, Trevor Hawkes dealt with three topics involving 'Linear Methods in Soluble Groups'. He first proved that if $H$ is an F-projector of $G$, where $F$ is a saturated formation, and if every irreducible character of $H$ can be extended to $G$, then $H$ has a normal complement. He then showed that the Fitting length of $G$ is bounded in terms of the composition length of a fischer subgroup (nilpotent injector). Finally he reported on groups with a fixed-point-free operator group.

Labhair Mícheál G'Searcóid faoi 'Comhaireamh p-Fhoghrúpaí'. David Lewis gave a talk on 'Sums of Squares in Division Algebras'.

We would like to thank the lecturers, the sponsors, and the participants for their continued support.

Rex Dark

\section{CONFERENCE ANNOUNCEMENT}

BAIL IV

The Fourth International Conference

on Boundary and Interior Layers-

\section{Computational and Asymptotic Methods}

$7-11$ July 1986

in

Novosibirsk, USSR

Hosted by the Siberian Branch of the

USSR Acaderny of Sciences

Chairman: Professor S.K. Godunov

Institute of Mathematics. Siberian Branch of the USSR Academy of Sciences. Novosibirsk, USSR

Co-Chairman: Professor J.J.H. Miller

Numerical Analysis Group. Trinity College, University of Dublin, Ireland

A formal First Announcement and Call for Papers will be made in due course by the Siberian Branch of the USSR Academy of Sciences in Novosibirsk.

Registration for participants from all non-socialist countries will be handled by the BAIL Secretariat in Dublin. Attendance from non-socialist countries is limited to a maximum of 80 participants, so early registration is advisable. If you are interested in attending, please contact the address below without delay. Participants from socialist countries should contact Novosibirsk directly.

Before BAIL IV you may attend the 10 th ICNMFD in BEIJING and then travel to NOvOSIBIRSK by rail via MONGOLIA. For more details read on:

The dates of the BAIL IV Conference have been harmonised with those of the 10 th International Conference on Numerical Methods 